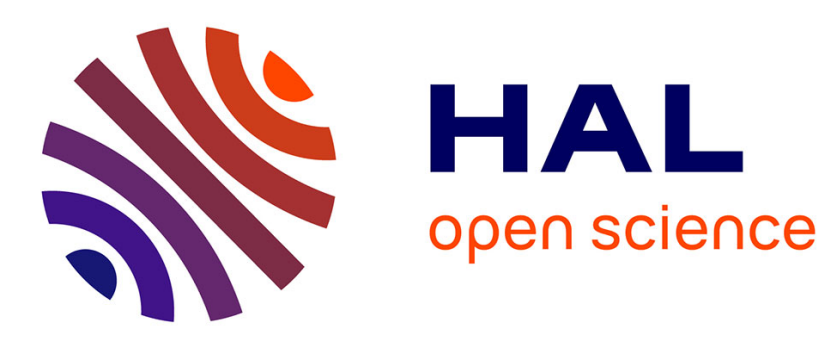

\title{
Modular randomized byzantine k-set agreement in asynchronous message-passing systems
}

\author{
Achour Mostefaoui, Hamouma Moumen, Michel Raynal
}

\section{To cite this version:}

Achour Mostefaoui, Hamouma Moumen, Michel Raynal. Modular randomized byzantine k-set agreement in asynchronous message-passing systems. The 17th International Conference on Distributed Computing and Networking (ICDCN'16), Jan 2016, Singapore, Singapore. pp.1-10. hal-02056378

\section{HAL Id: hal-02056378 https://hal.science/hal-02056378}

Submitted on 5 Mar 2019

HAL is a multi-disciplinary open access archive for the deposit and dissemination of scientific research documents, whether they are published or not. The documents may come from teaching and research institutions in France or abroad, or from public or private research centers.
L'archive ouverte pluridisciplinaire HAL, est destinée au dépôt et à la diffusion de documents scientifiques de niveau recherche, publiés ou non, émanant des établissements d'enseignement et de recherche français ou étrangers, des laboratoires publics ou privés. 


\title{
Modular Randomized Byzantine $k$-Set Agreement in Asynchronous Message-passing Systems
}

\author{
Achour Mostéfaoui $^{\dagger}$ Hamouma Moumen ${ }^{\ddagger}$ Michel Raynal $^{\star, \circ}$ \\ † LINA, Université de Nantes, 44322 Nantes Cedex, France \\ $\ddagger$ University of Bejaia, Algeria \\ * Institut Universitaire de France \\ ○ IRISA, Université de Rennes 35042 Rennes Cedex, France \\ Achour.Mostefaoui@univ-nantes.fr hamouma.moumen@gmail.com raynal@irisa.fr
}

June 29, 2015

\begin{abstract}
$k$-Set agreement is a central problem of fault-tolerant distibuted computing. Considering a set of $n$ processes, where up to $t$ may commit failures, let us assume that each process proposes a value. The problem consists in defining an algorithm such that each non-faulty process decides a value, at most $k$ dfferent values are decided, and the decided values satisfy some context-depending validity condition. Synchronous message-passing algorithms solving $k$-set agreement have been proposed for different failure models (mainly process crashes, and process Byzantine failures). Differently, $k$-set agreement cannot be solved in failure-prone asynchronous message-passing systems when $t \geq k$. To circumvent this impossibility an asynchronous system must be enriched with additional computational power.

Assuming $t \geq k$, this paper presents a distributed algorithm that solves $k$-set agreement in an asynchronous message-passing system wher up to $t$ processes may commit Byzantine failures. To that end, each process is enriched with randomization power. While randomized $k$-set agreement algorithms exist for the asynchronous process crash failure model where $t \geq k$, to our knowledge the proposed algorithm is the first that solves $k$-set agreement in the presence of up to $t \geq k$ Byzantine processes. Interestingly, this algorithm is signature-free, and ensures that no value proposed only by Byzantine processes can be decided by a non-faulty process. Its design is based on a modular construction which rests on a "no-duplicity" one-to-all broadcast abstraction, and two all-to-all communication abstractions.
\end{abstract}

Keywords: Asynchronous message-passing system, Broadcast abstraction, Byzantine process, Coin, Distributed algorithm, $k$-Set agreement, Randomized algorithm, Signature-free algorithm.

\section{Introduction}

Distributed agreement in the presence of process failures The world is distributed and more and more applications are now distributed. Moreover, when considering the core of non-trivial distributed applications, it appears that the computing entities (processes) have to agree in one way or another, for example to take a common decision, execute specific actions, or validate some commitment. Said another way, agreement problems lie at the core of distributed computing.

The most famous distributed agreement problem is the consensus problem. Let us consider a set of processes, where some of them may commit failures. Assuming each process proposes a value, the consensus problem is defined by the following properties: each non-faulty process must decide a value 
(termination), such that the same value is decided by the non-faulty processes (agreement), and this value satisfies some validity condition, which depends on the proposed values and the considered failure model [7, 17].

The $k$-set agreement problem is a natural weakening of consensus [6]. It allows the non-faulty processes to decide different values, as long as no more than $k$ values are decided. Hence, consensus is 1 -set agreement. Let us notice that $k$-set agreement can be easily solved in crash-prone systems where $k$ (the maximal number of different values that can be decided) is greater than $t$ (the maximal number of processes that may be faulty). The $k$-set agreement problem has applications, e.g., to compute a common subset of wavelengths (each process proposes a wavelength and at most $k$ of them are selected), or to duplicate $k$ state machines where at most one is required to progress forever [9, 22].

Byzantine failures This failure type has first been introduced in the context of synchronous distributed systems [12, 17, 20], and then investigated in the context of asynchronous distributed systems [1, 13, 21]. A process has a Byzantine behavior when it arbitrarily deviates from its intended behavior. We then say that it "commits a Byzantine failure" (otherwise we say the process is non-faulty or correct). This bad behavior can be intentional (malicious) or simply the result of a transient fault that altered the local state of a process, thereby modifying its behavior in an unpredictable way. Let us notice that process crashes (unexpected halting) define a strict subset of Byzantine failures.

Let us remind that (as already said) the world is distributed, asynchronous message-passing systems are more and more pervasive, processes have to agree in one way or another, and the assumption "no process has a bad behavior" is no longer sensible. Hence, agreement in asynchronous Byzantine message-passing systems is becoming a more and more important issue of fault-tolerance.

An impossibility result and how to cope with it Let consider a system made up of $n$ processes, where up to $t$ may be faulty. Whatever the value of $k$ (i.e., even if $k \leq t$ ), $k$-set agreement can always be solved if the system is synchronous [20]. The situation is different in asynchronous systems where $k$-set agreement is impossible to solve in the process crash failure model when $k \leq t[2,11,23]$. As Byzantine failures are more severe than process crash failures, this impossibility remains true in asynchronous Byzantine systems.

It follows from this impossibility that, when $k \leq t$, the underlying asynchronous distributed system has to be enriched with additional computational power for $k$-set agreement to be solved. Such an additional computational power can be provided with minimal synchrony assumptions (e.g., [3] which considers $k=1$ and Byzantine failures), appropriate failure detectors (e.g., [8] which considers $k=1$ and Byzantine failures), or randomization (e.g., [19] which considers $k=1$ and Byzantine failures, and $[5,15]$ which consider $k \leq t$ and crash failures, in read/write shared memory systems and messagepassing systems, respectively).

Intrusion-tolerant agreement with respect to Byzantine processes The validity property associated with a distributed agreement problem relates its outputs to its inputs. In a system where processes may commit Byzantine failures, there is no way to direct a Byzantine process to decide some specific value, and consequently the $k$-set agreement validity property can only be on the values decided by the correct processes.

A classical validity property for Byzantine agreement states that, when the non-faulty processes propose the same value, they must decide it. Hence, as soon as two non-faulty processes propose different values, any value can be decided by the correct processes, even a value "proposed" by a Byzantine process. (Let us observe that a Byzantine process can appear as proposing different values to different correct processes.) It follows that, as noticed and deeply investigated in [18], the solvability of Byzantine $k$-set agreement is sensitive to the particular validity property that is considered. 
In this paper we consider the following validity property (introduced in [16] where it is called intrusion-tolerance): no value proposed only by Byzantine processes can be decided by a non-faulty process. One way to be able to design a $k$-set algorithm providing this property, consists in allowing a non-faulty process to decide a default value $\perp$, except (to prevent triviality) when the non-faulty processes propose the same value. (The $\perp$ decision at some non-faulty processes can occur for example in the very adversary scenario where the non-faulty processes propose different values, while the Byzantine processes propose the same value). Another way to design a $k$-set algorithm providing intrusion-tolerance consists in adding a constraint on the total number of different values that can be proposed by the non-faulty processes. Let $m$ be this number. It is shown in [10] that, in a system of $n$ processes where up to $t$ processes may commit Byzantine failures, such a constraint is $n-t>m t$ (i.e., there is a value proposed by at least $(t+1)$ non-faulty processes).

Content of the paper This paper is on Byzantine $k$-set agreement. It has two main contributions.

- The first is a pair of all-to-all communication abstractions. The first one, called MV-broadcast (where MV stands for "Multivalued Validated"), allows the non-faulty processes to exchange values in such a way that all the non-faulty processes eventually obtain the same set of values, and none of these values is from Byzantine processes only. The second one, called SMV-broadcast (where S stands for "Synchronized") is built on top the first one, and is such that, if a non-faulty process obtains a set with a single value, the set obtained by any other non-faulty process contains this value. The important point is that these communication abstractions allow the processes to exchange values while eliminating the values sent only by Byzantine processes. They generalize to the "multivalue" case the communication abstractions introduced in [14], where the set of values that the processes exchange is limited to two values.

- The second contribution is a modular $k$-set agreement algorithm for asynchronous messagepassing systems where processes may commit Byzantine failures. This algorithm, which roundbased, relies on the previous SMV-broadcast abstraction, and (as already announced) on the additional computational power supplied by local random coins (with several sides). As far as we know, this is the first randomized $k$-set agreement algorithm for asynchronous Byzantine messagepassing systems.

The previous Byzantine-tolerant algorithms have two noteworthy features. The first is that they all are signature-free. This means that the "adversary" is not required to be computationally bounded. The second is their conceptual simplicity, which is a first-class property.

Roadmap The paper is composed of 5 sections. Section 2 presents the computation model and a basic broadcast abstraction (called ND-broadcast, where ND stand for "No Duplicity") introduced in [25]. As indicated by its name, this broadcast operation, which requires $t<n / 3$, allow to hide a duplicity behavior which can be produced by Byzantine processes. Then Section 3 presents the MV-broadcast and SMV-broadcast abstractions. SMV-broadcast is based on both MV-broadcast and ND-broadcast. Section 4 presents the modular randomized $k$-set agreement algorithm, whose construction relies on two instances of SMV-broadcast per round. Finally, Section 5 concludes the paper.

\section{Computation Model and ND-broadcast}

\subsection{Computation model}

Asynchronous processes The system is made up of a finite set $\Pi$ of $n>1$ asynchronous sequential processes, namely $\Pi=\left\{p_{1}, \ldots, p_{n}\right\}$. "Asynchronous" means that each process proceeds at its own pace, which may vary arbitrarily with time, and remains always unknown to the other processes. 
Communication network The processes communicate by exchanging messages through an asynchronous reliable point-to-point network. "Asynchronous" means that a message is eventually received by its destination process, i.e., there is no bound on message transfer delays. "Reliable" means that the network does not loss, duplicate, modify, or create messages. "Point-to-point" means that there is a bi-directional communication channel between each pair of processes. Hence, when a process receives a message, it can identify its sender.

A process $p_{i}$ sends a message to a process $p_{j}$ by invoking the primitive "send TAG $(m)$ to $p_{j}$ ", where TAG is the type of the message and $m$ its content. To simplify the presentation, it is assumed that a process can send messages to itself. A process receives a message by executing the primitive "receive()".

The operation broadcast $\operatorname{TAG}(m)$ is a macro-operation which stands for "for each $j \in\{1, \ldots, n\}$ send $\operatorname{TAG}(m)$ to $p_{j}$ end for". This operation is usually called unreliable broadcast (if the sender crashes in the middle of the for loop, it is possible that only an arbitrary subset correct processes receives a message).

Failure model Up to $t$ processes may exhibit a Byzantine behavior. A process that exhibits a Byzantine behavior is called faulty. Otherwise, it is correct or non-faulty. A Byzantine process is a process that behaves arbitrarily: it may crash, fail to send or receive messages, send arbitrary messages, start in an arbitrary state, perform arbitrary state transitions, etc. As a simple example, a Byzantine process, which is assumed to send a message $m$ to all the processes, can send a message $m_{1}$ to some processes, a different message $m_{2}$ to another subset of processes, and no message at all to the other processes. More generally, a Byzantine process has an unlimited computational power, and Byzantine processes can collude to "pollute" the computation. Let us notice that, as each pair of processes is connected by a channel, no Byzantine process can impersonate another process, but Byzantine processes are not prevented from influencing the delivery order of messages sent to correct processes.

Discarding messages from Byzantine processes If, according to its algorithm, a process $p_{j}$ is assumed to send a single message TAG () to a process $p_{i}$, then $p_{i}$ processes only the first message $\operatorname{TAG}(v)$ it receives from $p_{j}$. This means that, if $p_{j}$ is Byzantine and sends several messages $\operatorname{TAG}(v), \operatorname{TAG}\left(v^{\prime}\right)$ where $v^{\prime} \neq v$, etc., all of them except the first one are discarded by their receivers. (Let us observe that this does not prevent multiple copies of the first message $\operatorname{TAG}()$ to be received and processed by their receiver.)

Notation This computation model is denoted $\mathcal{B} \mathcal{A M} \mathcal{P}_{n, t}[\emptyset]$ (BAMP stands for "Byzantine Asynchronous Message Passing"). In the following, this model is both restricted with a constraint on $t$ and enriched with an object providing processes with additional computational power. More precisely, $\mathcal{B A M P}_{n, t}[t<n / \alpha]$ (where $\alpha$ is a positive integer) denotes the model $\mathcal{B A M P}_{n, t}[\emptyset]$ where the maximal number of faulty processes is smaller than $n / \alpha$, and $\mathcal{B A M P} \mathcal{P}_{n, t}[t<n / \alpha, \mathrm{LRC}]$ denotes the model $\mathcal{B A M P}{ }_{n, t}[t<n / \alpha]$ where each process is enriched with a local random coin (LRC). Let us notice that, as LRC belongs to the model, it is given for free in $\mathcal{B A M P} \mathcal{P}_{n, t}[t<n / \alpha, \mathrm{LRC}]$.

Time complexity When computing the time complexity we ignore local computation time, and consider the longest sequence of causally relate messages $m_{1}, m_{2}, \ldots, m_{z}$ (i.e., for any $x \in[2 . . z]$, the reception of $m_{x-1}$ is a requirement for the sending of $m_{x}$ ). The size of such a longest sequence defines the time complexity. 


\subsection{No-duplicity broadcast}

Definition of ND-broadcast The ND-broadcast communication abstraction was introduced by S. Toueg in [25]. It is defined by two operations denoted ND_broadcast() and ND_deliver(), which allow the processes to eliminate bad behaviors of Byzantine processes. More precisely, a Byzantine process is prevented from sending different messages to different correct processes, while it is assumed to send the very same message to all of them.

When a process invokes ND_broadcast() we say that it "ND-broadcasts" a message, and when it invokes ND_deliver() we say that it "ND-delivers" a message 1 . Considering an instance of ND-broadcast where the operation ND_broadcast() is invoked by a process $p_{i}$, this communication abstraction is defined by the following properties.

- ND-Validity. If a non-faulty process ND-delivers a message from $p_{i}$, then, if it is non-faulty, $p_{i}$ ND-broadcast this message.

- ND-No-duplicity. No two non-faulty processes ND-deliver distinct messages from $p_{i}$.

- ND-Termination. If the sender $p_{i}$ is non-faulty, all the non-faulty processes eventually ND-deliver its message.

Let us observe that, if the sender $p_{i}$ is faulty, it is possible that some non-faulty processes NDdeliver a message from $p_{i}$ while others do not ND-deliver a message from $p_{i}$. As already indicated, the no-duplicity property prevents non-faulty processes from ND-delivering different messages from a faulty sender.

An algorithm implementing ND-broadcast It is shown in [25] that $t<n / 3$ is a necessary requirement to implement ND-broadcast in a Byzantine asynchronous message-passing system. Algorithm 1 (from [25]) implements ND-broadcast in $\mathcal{B A M P}_{n, t_{n, t}}[t<n / 3]$ as follows.

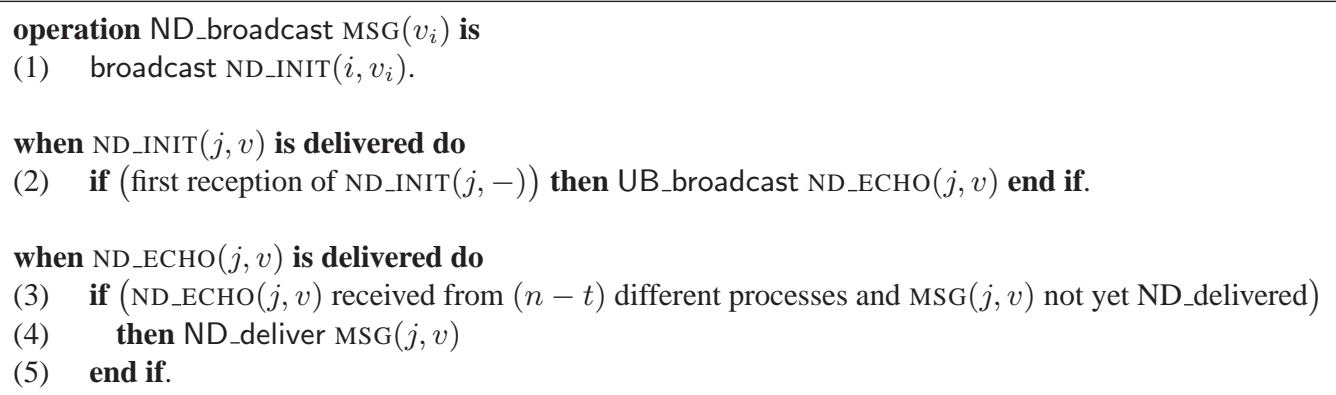

Algorithm 1: Implementing ND-broadcast in $\mathcal{B A M P}_{n, t}[t<n / 3]$

When a process $p_{i}$ wants to ND-broadcast a message whose content is $v_{i}$, it broadcasts the message $\operatorname{ND} \_I N I T\left(i, v_{i}\right)$ (line 1$)$. When a process receives a message $\operatorname{ND} \operatorname{INIT}(j,-)$ for the first time, it broadcasts a message ND_ECHO $(j, v)$ where $v$ is the data content of the ND_INIT () message (line 2). If the message ND_INIT $(j, v)$ received is not the first message $\operatorname{ND\_ INIT}(j,-), p_{j}$ is Byzantine and the message is discarded. Finally, when $p_{i}$ has received the same message $\operatorname{ND} \_\operatorname{ECHO}(j, v)$ from $(n-t)$ different processes, it locally ND-delivers $\operatorname{MSG}(j, v)$ (lines 3-4).

The algorithm considers an instance of ND-broadcast, i.e., a correct process invokes at most once ND-broadcast. Adding a sequence number to each message allows each process to ND-broadcast a sequence of messages.

Theorem 1. Algorithm 1 implements ND-broadcast in the system model $\mathcal{B A M P}{ }_{n, t}[t<n / 3]$.

\footnotetext{
${ }^{1}$ A similar vocabulary will be used for the abstractions MV-broadcast and SMV-broadcast introduced in Section 3.
} 
Proof To prove the ND-termination property, let us consider a non-faulty process $p_{i}$ that ND-broadcasts the message $\operatorname{MSG}\left(v_{i}\right)$. As $p_{i}$ is non-faulty, the message ND_INIT $\left(i, v_{i}\right)$ is received by all the non-faulty processes, which are at least $(n-t)$, and every non-faulty process broadcasts ND_ECHO $\left(i, v_{i}\right)$ (line 2$)$. Hence, each non-faulty process receives the message ND_ECHO $\left(i, v_{i}\right)$. from $(n-t)$ different processes. It follows that every non-faulty process eventually ND-delivers the message MSG $\left(i, v_{i}\right)$ (lines 3-4).

To prove the ND-no-duplicity property, let us assume by contradiction that two non-faulty processes $p_{i}$ and $p_{j}$ ND-deliver different messages $m_{1}$ and $m_{2}$ from some process $p_{k}$ (i.e., $m_{1}=\operatorname{MSG}(k, v)$ and $m_{2}=\operatorname{MSG}(k, w)$, with $\left.v \neq w\right)$. It follows from the predicate of line 3, that $p_{i}$ received $\operatorname{ECHO}(k, v)$ from a set of $(n-t)$ distinct processes, and $p_{j}$ received $\operatorname{ECHO}(k, w)$ from a set of $(n-t)$ distinct processes. As $n>3 t$, it follows that the intersection of these two sets contains a non-faulty process. But, as it is non-faulty, this sent the same message ND_ECHO() to $p_{i}$ and $p_{j}$ (line 2). Hence, $m_{1}=m_{2}$, which contradicts the initial assumption.

To prove the ND-validity property, we show that, if Byzantine processes forge and broadcast a message ND_ECHO $(i, w)$ such that $p_{i}$ is correct and has never invoked ND_broadcast MSG $(w)$, then no correct process can ND-deliver $\operatorname{MSG}(i, w)$. Let us observe that at most $t$ processes can broadcast the message ND_ECHO $(i, w)$. As $t<n-t$, it follows that the predicate of line 3 can never be satisfied at a correct process. Hence, if $p_{i}$ is correct, no correct process can ND-deliver from $p_{i}$ a message that was not been ND-broadcast by $p_{i}$.

$\square_{\text {Theorem } 1}$

It is easy to see that this implementation uses two consecutive communication steps and $O\left(n^{2}\right)$ underlying messages ( $n-1$ in the first communication step, and $n(n-1)$ in the second one). Moreover, there are two types of protocol messages, and the size of the control information added to a message is $\log _{2} n$ (sender identity).

\section{Multivalued Validated Broadcast: MV-broadcast and SMV-broadcast}

This section presents the all-to-all MV-broadcast and SMV-broadcast communication abstractions. "Allto-all" mean that it is assumed that all the non-faulty processes invoke the corresponding broadcast operation. As indicated in the introduction, these abstractions extend to the "multivalue" case the BVbroadcast and SBV-broadcast communication abstractions introduced in [14], which consider binary values only.

\subsection{Multivalued validated all-to-all broadcast}

Definition of MV-broadcast This communication abstraction provides the processes with a single operation denoted MV_broadcast () . When a process invokes $\mathrm{MV}_{-}$broadcast TAG $(m)$, we say that it "MV-broadcasts the message typed TAG and carrying the value $m$ ". The invocation of MV_broadcast $\mathrm{TAG}(m)$ does not block the invoking process. The aim of MV-broadcast is to eliminate the values (if any) that have been broadcast only by Byzantine processes.

In an MV-broadcast instance, each correct process $p_{i}$ MV-broadcasts a value and eventually obtains a set of values. To store these values, MV-broadcast provides each process $p_{i}$ with a read-only local variable denoted mv_values $i$. This set variable, initialized to $\emptyset$, increases asynchronously when new values are received.

Definition Each instance of MV-broadcast is defined by the four following properties.

- MV-Termination. The invocation of MV_broadcast() by a correct process terminates.

- MV-Justification. If $p_{i}$ is a correct process and $v \in m v_{-} v a l i d_{i}, v$ has been MV-broadcast by a correct process. 
- MV-Uniformity. If $p_{i}$ is a correct process and $v \in m v_{-} v a l i d_{i}$, eventually $v \in m v_{-} v a l i d_{j}$ at every correct process $p_{j}$.

- MV-Obligation. Eventually the set $m v_{-} v a l i d_{i}$ of each correct process $p_{i}$ is not empty.

The following properties are immediate consequences of the previous definition.

- MV-Equality. The sets $m v_{-} v a l i d_{i}$ of the correct processes are eventually non-empty and equal.

- MV-Integrity. The set $m v_{-} v a l i d_{i}$ of a correct process $p_{i}$ never contains a value MV-broadcast only by Byzantine processes.

Feasibility condition in the presence of up to $t$ Byzantine processes Let $m$ be the number of different values MV-broadcast by correct processes. It follows from the previous specification that, even when the (at most) $t$ Byzantine processes propose the same value $w$, which is not proposed by correct processes, $w$ cannot belong to the set $m v_{-} v a l i d_{i}$ of a correct process $p_{i}$. This can be ensured if and only if there is a value MV-broadcast by at least $(t+1)$ correct processes. This feasibility condition is captured by the predicate $n-t>m t$ (a proof of this feasibility condition can be found in [10]). Hence $n>(m+1) t$ is a feasibility condition for MV-broadcast to cope with up to $t$ Byzantine processes. Let us notice that, as $m \geq 2, n>(m+1) t$ implies $n>3 t$.

An MV-broadcast algorithm Algorithm 2 describes a simple implementation of MV-broadcast, suited to the system model $\mathcal{B} \mathcal{A M P} \mathcal{P}_{n, t}[t<n /(m+1)]$. This algorithm is based on a simple "echo" mechanism. Differently from previous echo-based algorithms (e.g., $[4,24])$, the echo is used here with respect to each value that has been received (whatever the number of processes that broadcast it), and not with respect to each pair composed of a value plus the identity of the process that broadcast this value. Hence, a value entails at most one echo per process, whatever the number of processes that MV-broadcast this value.

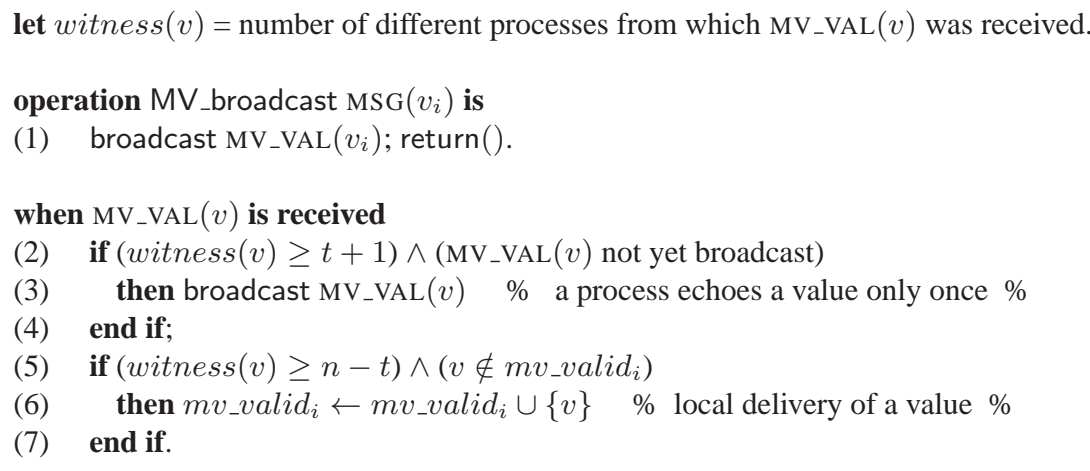

Algorithm 2: Implementing MV-broadcast in $\mathcal{B A M P}_{n, t}[t<n /(m+1)]$

When a process $p_{i}$ invokes MV_broadcast MSG $\left(v_{i}\right)$, it broadcasts $\operatorname{MV} \_\operatorname{VAL}\left(v_{i}\right)$ to all the processes (line 1). Then, when a process $p_{i}$ receives (from any process) a message MV_VAL $(v)$, (if not yet done) it forwards this message to all the processes (line 3 ) if it has received the same message from at least $(t+1)$ different processes (line 2). Moreover, if $p_{i}$ has received $v$ from at least $(2 t+1)$ different processes, the value $v$ is added to $m v \_v a l i d_{i}$ (lines 5-6). Let us notice that, except in the case where $\left|m_{v} v_{-} v a l i d_{i}\right|=m$, no correct process $p_{i}$ can know if its set mv_valid $_{i}$ has obtained its final value.

Theorem 2. Algorithm 2 implements $M V$-broadcast in the system model $\mathcal{B A M P}{ }_{n, t}[t<n /(m+1)]$.

Proof The proof of the MV-Termination property is trivial. If a correct process invokes MV_broadcast () , it eventually sends a message to each process, and terminates. 
Proof of the MV-Justification property. To show this property, we prove that a value MV-broadcast only by faulty processes cannot be added to the set mv_valid $d_{i}$ of a correct process $p_{i}$. Hence, let us assume that only faulty processes MV-broadcast $v$. It follows that a correct process can receive the message $\operatorname{MV} \_\operatorname{VAL}(v)$ from at most $t$ different processes. Consequently the predicate of line 2 cannot be satisfied at a correct process. Moreover, as $n-t>t$, the predicate of line 5 cannot be satisfied either at a correct process, and the property follows.

Proof of the MV-Uniformity property. If a value $v$ is added to the set $m_{-} v_{-} v a l i d_{i}$ of a correct process $p_{i}$ (local delivery), this process received $\operatorname{MV} \_\operatorname{VAL}(v)$ from at least $(n-t)$ different processes (line 5), i.e., from at least $(n-2 t)$ different correct processes. As each of these correct processes sent this message to all the processes, it follows that the predicate of line 2 is eventually satisfied at each correct process, which consequently broadcasts $\operatorname{MV}_{-} \operatorname{VAL}(v)$ to all. As there are at least $(n-t)$ correct processes, the predicate of line 5 is then eventually satisfied at each correct process, and the MV-Uniformity property follows.

Proof of the MV-Obligation property. It follows from he feasibility condition $n>(m+1) t$, that there is a value $v$ MV-broadcast by at least $(t+1)$ correct processes. It then follows that these processes issue MV_broadcast MSG $(v)$, and consequently all correct processes first deliver the message MV_VAL $(v)$ and then broadcast at line 3 (if not previously done). Hence, each correct process $p_{i}$ eventually delivers this message from $(n-t)$ processes and and adds $v$ to its set $m_{-} v_{v a l i d}$ (line 5-6), which proves the property.

Cost of the algorithm As at most $m$ values are MV-broadcast by the correct processes, it follows from the text of the algorithm that each correct process broadcasts each of these values at most once (at line 1 or line 3). Hence, if there are $c \in[n-t . . n]$ correct processes, their broadcasts entail the sending of at most $m c n$ messages MV_VAL( $)$. Finally, whatever the number of values that are MV-broadcast, the algorithm requires at most two communication steps.

\subsection{Synchronized multivalued validated all-to-all broadcast}

Definition of SMV-broadcast This all-to-all communication abstraction provides the processes with a single operation denoted SMV_broadcast $($ ). As indicated by its name, its aim is to synchronize processes so that, if a single value $v$ is delivered to a correct process, then $v$ is delivered to all the correct processes.

In an SMV-broadcast instance, each correct process invokes SMV_broadcast TAG $(m)$, where TAG is the type of the message and $m$ value it wants to broadcast. Such an invocation returns to the invoking process $p_{i}$ a set denoted $v_{i e w}$ and called a local view. We say that a process contributes to a set view if the value it SMV-broadcasts belongs to view $w_{i}$. SMV-broadcast is defined by the following properties.

- SMV-Termination. The invocation of SMV_broadcast TAG() by a correct process terminates.

- SMV-Obligation. The set $v i e w_{i}$ returned by a correct process $p_{i}$ is not empty.

- SMV-Justification. If $p_{i}$ is correct and $v \in v i e w_{i}$, then a correct process SMV-broadcast $v$.

- SMV-Inclusion. If $p_{i}$ and $p_{j}$ are correct processes and view $w_{i}=\{v\}$, then $v \in v i e w_{j}$.

- SMV-Contribution. If $p_{i}$ is correct, at least $(n-t)$ processes contribute to its set view $w_{i}$.

- SMV-No-duplicity. Let VIEW be the union of the sets view $w_{i}$ of the correct processes. A process contributes to at most one value of VIEW.

The following property is an immediate consequence of the previous definition. property.

- SMV-Singleton. If $p_{i}$ and $p_{j}$ are correct, $\left[\left(\right.\right.$ view $\left.\left._{i}=\{v\}\right) \wedge\left(v_{i e w}=\{w\}\right)\right] \Rightarrow(v=w)$.

Let $v \in V I E W, p_{i}$ a correct process, and $p_{j}$ a Byzantine process. It is possible that, while the value $v$ was SMV-broadcast by $p_{i}$ (hence $p_{i}$ contributed to VIEW), $p_{j}$ also appears as contributing to VIEW with the same value $v$. The SMV-No-duplicity property states the following: no value $w \in V I E W \backslash\{v\}$ appears as a contribution of $p_{j}$. 
An SMV-broadcast algorithm Algorithm 3 implements SMV-broadcast in $\mathcal{B A M P}_{n, t}[t<n /(m+$ 1)]. A process $p_{i}$ first MV-broadcasts a message MSG $\left(v_{i}\right)$ and waits until the associated set mv_values $_{i}$ is not empty (lines 1-2). Let us remind that, when $p_{i}$ stops waiting, mv_values $_{i}$ has not necessarily obtained its final value. Then, $p_{i}$ extracts a value $w$ from $m_{-} v_{-} v a l u e s_{i}$ and ND-broadcasts it to all (line 3). Let us notice that, due to the ND-no-duplicity property, no two correct processes can NDdeliver different values from the same Byzantine process.

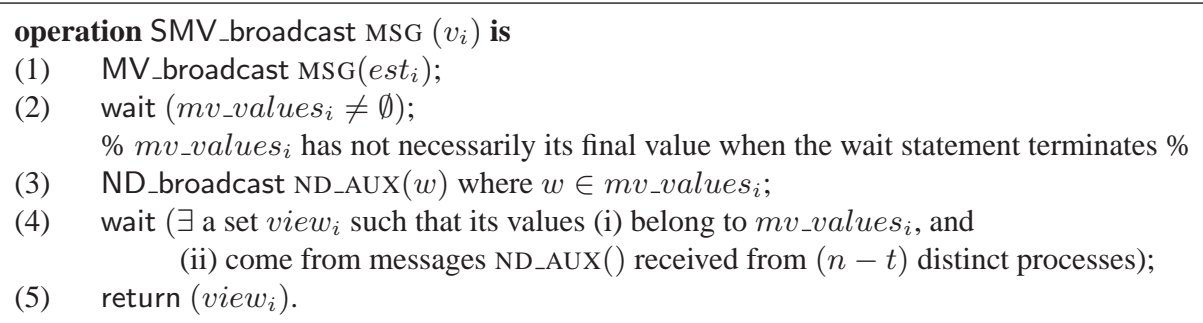

Algorithm 3: Implementing SMV-broadcast in $\mathcal{B A M P}_{n, t}[t<n /(m+1)]$

Finally, $p_{i}$ waits until the predicate of line 4 is satisfied. This predicate has two aims. The first is to discard from view $w_{i}$ (the set returned by $p_{i}$ ) a value broadcast only by Byzantine processes. Hence the predicate $v_{i e w} \subseteq$ mv $_{-} v_{\text {values }}$. The second aim is to ensure that, if the view view $w_{i}$ of a correct process $p_{i}$ contains a single value, then this value eventually belongs to the view view $w_{j}$ of any correct process $p_{j}$. To this end, $(n-t)$ different processes (hence, at least $(n-2 t)$ correct processes) must contribute to view $_{i}$.

Multiset version of SMV-broadcast While a value belongs or does not belong to a set, a multiset (also called a bag) is a set in which the same value can appear several times. As an example, while $\{a, b, c\}$ and $\{a, b, b, c, c, c\}$ are the same set, they are different multisets.

It is easy to see that the "set" version of the SMV-broadcast (where view $w_{i}$ is a set) and Algorithm 3 can be easily converted into a "multiset" version where view $w_{i}$ is a multiset. Both versions will be used in the randomized $k$-set agreement presented in Section 4.

Theorem 3. Algorithm 3 implements SMV-broadcast in the system model $\mathcal{B A M P} \mathcal{P}_{n, t}[t<n /(m+1)]$.

Proof Proof of the SMV-Termination property. Let us first observe that, due to the MV-Termination property and the MV-Obligation property of the underlying MV-broadcast, no correct process blocks forever at line 2. As there are at least $(n-t)$ correct processes, and none of them blocks forever a line 2, it follows from the ND-Termination property that each correct process return from the ND-broadcast at line 3, and eventually ND-delivers values from at least the $(n-t)$ correct processes. Moreover, due to the MV-Justification property, these values have been SMV-broadcast by correct processes, and, due to the MV-Uniformity property, the sets $m_{-} v_{-} v a l i d_{i}$ of all correct processes are eventually equal. It then follows that the predicate of line 4 becomes eventually satisfied at any correct process $p_{i}$, and consequently the invocations of SMV_broadcast () of the correct processes terminate.

Proof of the SMV-Obligation property. Any correct process $p_{i}$ eventually ND-delivers $(n-t)$ messages ND_AUX () sent by correct processes. As (a) these messages carry values taken from the set mv_values $s_{x}$ of correct processes, and (b) these sets (b.1) are eventually equal at all correct processes, and (b.2) contain all values ND-broadcast at line 3 by the correct processes, it follows (from the predicate of line 4) that the set $v_{i e w_{i}}$ returned by a correct process is not empty.

Proof of the SMV-Justification property. This property follows directly from the fact that the predicate of line 4 discards the values ND-broadcast only by Byzantine processes, and from the MV-Justification property, namely, the set $m v_{-} v a l u e s_{i}$ of a correct process contains only values MV-broadcast by correct processes. 
Proof of the SMV-Inclusion property. Let us consider a correct process $p_{i}$ and assume view $w_{i}=\{v\}$. It follows from the predicate of line 4 that $p_{i}$ has ND-delivered the same message ND_AUX $(v)$ from at least $(n-t)$ different processes. As at most $t$ of them are Byzantine, it follows that $p_{i}$ ND-delivered this message from at least $(n-2 t)$ different correct processes, i.e., as $n-2 t \geq t+1$, from at least $(t+1)$ correct processes.

Let us consider any correct process $p_{j}$. This process ND-delivered messages ND_AUX () from at least $(n-t)$ different processes. As $(n-t)+(t+1)>n$, it follows that there is a correct process $p_{x}$ that ND-broadcast the same message ND_AUX $(v)$ to $p_{i}$ and $p_{j}$. It follows that $v \in v i e w_{j}$, which concludes the proof of the lemma.

Proof of the SMV-Contribution property. This property follows trivially from the part (ii) of the waiting predicate of line 4.

Proof of the SMV-No-duplicity property. This property is an immediate consequence of the ND-Noduplicity property of the ND-broadcast issued at line 3.

$\square_{\text {Theorem } 3}$

\section{Randomized Byzantine $k$-Set Agreement}

This section presents and proves correct a Byzantine $k$-set agreement algorithm, which is modularly built on top of the SMV-broadcast communication abstraction and the additional computational power supplied to each process by a local random coin (LRC).

\subsection{Intrusion-tolerant Byzantine $k$-set agreement}

The intrusion-tolerant Byzantine (ITB) $k$-set agreement was informally presented in the introduction. It is assumed that each non-faulty process invokes an operation called propose ${ }_{k}()$. This operation has an input parameter $v$, which is the value proposed by the invoking process. It returns a value, which is called the "value decided" by the invoking process. ITB $k$-set agreement is formally defined in terms of properties that any solution must to satisfy. When considering deterministic $k$-set agreement algorithms, these properties are the following ones.

- KS-D-Termination. If a correct process invokes $\operatorname{propose}_{k}()$, it decides a value.

- KS-Validity. If a correct process decides $v$, then $v$ was proposed by a correct process.

- KS-Agreement. The set of values decided by the correct processes contains at most $k$ values.

As we are interested in a randomized algorithm to solve $k$-set agreement, the termination property has to be weakened as follows: any correct process decides with probability 1 . In the context of roundbased randomized algorithms, this property can restated as follows.

- KS-RR-Termination. $\lim _{r \rightarrow+\infty}\left(\right.$ Probability $\left[p_{i}\right.$ decides by round $\left.\left.r\right]\right)=1$.

\subsection{Enriching the basic Byzantine asynchronous model with a random coin}

As announced, the additional computational power used to solve ITB $k$-set agreement despite Byzantine processes is supplied by a multi-sided random coin denoted LRC. The random abstraction LRC provides each process with a local coin that provides it with a single operation denoted random(). Each invocation takes a finite set $X$ as input parameter, and returns a value of $X$ such that each value of $X$ as the probability $1 /|X|$ to be returned.

As seen in the introduction, we assume $k \leq t$. Moreover, we have also seen that, in order a correct process decides neither a value proposed only by Byzantine processes, nor a predefined default value, it is assumed that, whatever the domain of the values that can be proposed by the correct processes, in any execution, at most $m$ different values are proposed by correct processes, where $m$ depends on $n$ and $t$, 
namely, $n>m(t+1)$. Finally, to rule out the trivial algorithm in which a correct process decides the value it proposes, we assume $k<m$.

Hence, assuming the non-triviality conditions $k<m$ and $k \leq t$, and the fact that, in any execution, at most $m$ different values are proposed by the correct processes, the system model considered here to solve the ITB $k$-set agreement problem is $\mathcal{B A M P}_{n, t}[t<n /(m+1), \mathrm{LRC}]$.

\subsection{A randomized Byzantine $k$-set agreement algorithm}

Local variables To solve the ITB $k$-set agreement problem, Algorithm 4, which is round-based, relies on a very modular construction. Each process $p_{i}$ manages two local variables whose scope is the whole execution: a local round number $r_{i}$, and a local estimate of a decision value, denoted $e s t_{i}$. It also manages three local variables whose scope is the current round $r$ : a multiset view $w_{i}[r, 1]$, an auxiliary variable aux, and a set view $_{i}[r, 2]$.

Description of the algorithm When $p_{i}$ invokes propose ${ }_{k}\left(v_{i}\right)$ it assigns $v_{i}$ to $e s t_{i}$ and initializes $r_{i}$ to 0 (line 1). Then $p_{i}$ enters a loop that it will exit at line 8 by executing return $(v)$, which returns the decided value $v$ and stops its participation in the algorithm.

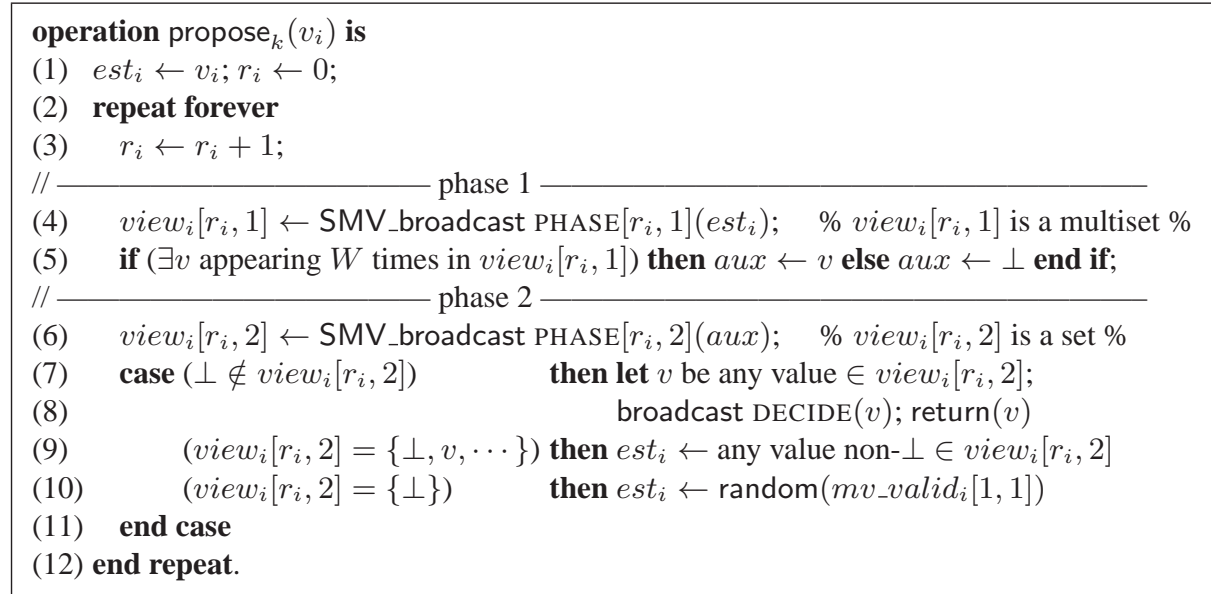

Algorithm 4: Byzantine $k$-set agreement based on SMV-broadcast, and local random coins

Each round $r$ executed by a process $p_{i}$ is made up of two phases. During the first phase of round $r$, each correct process $p_{i}$ invokes SMV_broadcast $\left(e s t_{i}\right)$ (multiset version) and stores the multiset returned by this invocation in view $i[r, 1]$. Let us remind that this multiset contains only values SMV-broadcast by at least one correct process. The aim of this phase is to build a global set ${ }^{2}$, denoted $A U X[r]$, which contains at most $(k+1)$ values, such that at most $k$ of them are contributed by correct processes, and the other one is the default value $\perp$. To this end, each correct process $p_{i}$ checks if there is a value $v$ that appears "enough" (say $W$ ) times in the multiset view $w_{i}[r, 1]$. If there is such a value $v, p_{i}$ adopts it (assignment aux $\leftarrow v$ ), otherwise it adopts the default value $\perp$ (line 5).

The set $A U X[r]$ is made up of the aux variables of all the correct processes. For $A U X[r]$ to contain at most $k$ non- $\perp$ values, $W$ has to be such that $(k+1) W>n$ (there are not enough processes for $(k+1)$ different values such that each of them was contributed by $W$ processes $)^{3}$. Hence, $W>n /(k+1)$.

When it starts the second phase of round $r$, each correct process $p_{i}$ invokes SMV_broadcast $($ aux $)$ (set version) and stores the set it obtains in view $i r, 2]$. Due to the properties of SMV-broadcast,

\footnotetext{
${ }^{2}$ While the value of this set could be known by an external global observer, its value can never be explicitly known by a correct process. However, a process can locally build an approximation of it during the second phase, see below.

${ }^{3}$ Let us remind that, due to the ND-broadcast used in the algorithm implementing SMV-broadcast, two correct processes cannot ND-deliver different values from the same Byzantine process.
} 
view $_{i}[r, 2]$ is a local approximation of $A U X[r]$, namely, view $i[r, 2] \subseteq A U X[r]$. Then, the behavior of $p_{i}$ depends on the content of the set view $\left.i r, 2\right]$.

- If $\perp \notin$ view $_{i}[r, 2], p_{i}$ decides any value in view $i[r, 2]$ (lines 7-8).

- If view $w_{i}[r, 2]$ contains $\perp$ and non- $\perp$ values, $p_{i}$ updates its current estimate $e s t_{i}$ to any non- $\perp$ value of view $_{i}[r, 2]$ and starts new round (line 9).

- If view $w_{i}[r, 2]$ contains only $\perp, p_{i}$ starts a new round, but updates previously its current estimate $e s t_{i}$ to a random value (line 10). This random value is obtained from the set (denoted mv_valid $_{i}[1,1]$ in the algorithm) locally output by the first MV-broadcast instance invoked by $p_{i}$. The use of these sets allows the algorithm to benefit from the fact that these sets are eventually equal at all correct processes (MV-Equality property). The KS-Termination relies on this property.

As shown in the proof, an important behavioral property of the algorithm lies in the fact that, at any round $r$, it is impossible for two correct processes $p_{i}$ and $p_{j}$ to be such that $(\perp \notin$ view $i[r, 2]) \wedge\left(\right.$ view $_{i}[r, 2]=$ $\{\perp\})$. These two predicates are mutually exclusive.

On the value of $W \quad$ The value $W$ is used at line 5 for a safety reason, namely, no more than $k$ non- $\perp$ values can belong to the set $A U X[r]$. As we have seen, this is captured by the constraint $W(k+1)>n$. It appears that $W$ has also to be constrained for a liveness reason, namely, when the correct processes start a new round $r$ with at most $k$ different estimates values, none of them must adopt the value $\perp$ at line 5 (otherwise, instead of deciding at line 7, they could loop forever).

This liveness constraint is as follows. Let us consider the size of the multiset view $w_{i}[r, 1]$ obtained at line 4. In the worst case, when the correct processes start a new round $r$ with at most $k$ different estimates, view $i[r, 1]$ may contain $(k-1)$ different values, each appearing $(W-1)$ times, and only one value that appears $W$ times. Hence, view $i[r, 1]$ must contain at least $(W-1)(k-1)+W=(W-1) k+1$ elements. As it follows from Algorithm 3 that $\mid$ view $_{i}[r, 1] \geq n-t$, we obtain the liveness constraint $n-t \geq(W-1) k+1$.

On message identities The messages PHASE() SVM-broadcast at line 4 and line 6 are identified by a pair $[r, x]$ where $r$ is a round number and $x \in\{1,2\}$ a phase number. Each of these messages gives rise to underlying messages ND_AUX () (Algorithm 2), MV_VAL () (Algorithm 1), and underlying sets witness() (Algorithm 1). Each of them inherits the pair identifying the message PHASE() it originates from.

On the messages $\operatorname{DECIDE}()$ Before a correct process decides a value $v$, it sends a message $\operatorname{DECIDE}(v)$ to each other process (line 8). Then, it stops its execution. This halting has not to prevent correct processes from terminating, which could occur if they wait forever underlying messages ND_AUX () or MV_VAL() from $p_{i}$.

To this end, a message $\operatorname{DECIDE}(v)$ has to be considered as representing an infinite set of messages. More precisely if, while executing a round $r$, a process $p_{i}$ receives a message $\operatorname{DECIDE}(v)$ from a process $p_{j}$, it considers that it has received from $p_{j}$ the following set of messages: $\left\{\right.$ ND_AUX $\left[r^{\prime}, 1\right](v)$, ND_AUX $\left.\left[r^{\prime}, 2\right](v), \operatorname{MV} \_V A L\left[r^{\prime}, 1\right](v), \operatorname{MV} \_V A L\left[r^{\prime}, 2\right](v)\right\}_{r^{\prime} \geq r}$. It is easy to see that the messages DECIDE () simulate a correct message exchange that could be produced, after it has decided, by a deciding but nonterminating process.

Another solution would consist in using a Reliable Broadcast abstraction that copes with Byzantine processes. In this case, a process could decide a value $v$ as soon as it has RB-delivered $(t+1)$ messages $\operatorname{DECIDE}(v)$. An algorithm implementing such a reliable broadcast is presented in [4]. This algorithm requires $O\left(n^{3}\right)$ messages and assumes $n<t / 3$, which is a necessary requirement to implement reliable broadcast in the presence of Byzantine processes. 


\subsection{Proof of the algorithm}

The proof considers the system model $\mathcal{B A M P}_{n, t}[t<n /(m+1), \mathrm{LRC}]$, the algorithmic safety and liveness constraints on $W$, namely, $W(k+1)>n$ and $n-t \geq(W-1) k+1$, and the non-triviality condition $(k<m) \wedge(k \leq t)$.

Preliminary remark 1 The proof considers the semantic of the messages DECIDE () described previously. This is equivalent to consider that, after it has decided, a correct process continues executing while skipping line 8 .

Notation Given a round $r$, let $E S T[r]$ be the set of estimate values of the correct processes when they start round $r$, and $A U X[r]$ be the set including the values of the $a u x_{i}$ variables of the correct processes at the end of the first phase of round $r$ (i.e., just after line 5). let us notice that $A U X[r]$ can contain $\perp$.

Preliminary remark 2 The proof of the MV-Obligation property requires that at most $m$ different values are MV-broadcast. Hence, this requirement extends to the invocations SMV_broadcastPHASE $[r, x]()$, where $x \in\{1,2\}$. By assumption, this requirement is initially satisfied, namely, $|E S T[1]| \leq m$. We will see in the proof that (i) $A U X[r]$ contains at most $k$ values proposed by correct processes plus possibly $\perp$, (ii) view $i[r, 2]$ is a subset of $A U X[r]$, and (iii) mv_valid $_{i}[1,1]$ contains only values proposed by correct processes. From the previous observations we conclude that at most $m$ different values are SMV-broadcast at line 4 and line 6 of Algorithm 4.

Lemma 1. If a correct process decides a value, this value was proposed by a correct process.

Proof Let us consider the first round $r=1$. It follows from the MV-Justification property of the SMVbroadcast invocation at line 4 that the multiset view $w_{i}[1,1]$ of any correct process $p_{i}$ contains only values SMV-broadcast by correct processes. The same is true for the set view $w_{i}[1,2]$ which, in addition, can also contain the default value $\perp$. It follows that, if a correct process decides at lines 7-8, it decides a value proposed by a correct process. If a correct process progresses to the next round, it executes line 9 or line 10 (for line 10, this follows from the MV-Justification property of the of the MV-broadcast generated by the invocation SMV_broadcast PHASE $[1,1]\left(e s t_{i}\right)$ ). In both cases, its new estimate value is a value proposed by a correct process. Hence the estimate values of the processes that start the second round are values proposed by correct processes. Applying this reasoning to the sequence of rounds, it follows that no correct process can decide a value not proposed by a correct process.

$\square_{\text {Lemma } 1}$

Lemma 2. AUX $[r]$ contains at most $k$ non- $\perp$ values, plus possibly the default value $\perp$.

Proof Let us assume that $A U X[r]$ contains $(k+1)$ non- $\perp$ values. If a value belongs to this set, it is the value of the local variable $a u x_{i}$ of a correct process $p_{i}$, which appears at least $W$ times in the multiset view $_{i}[r, 1]$ (line 5). Moreover, due to SMV-No-duplicity property, a process (correct or Byzantine) contribute to at most one of these values. It follows from these observations that, if $A U X[r]$ contains $(k+1)$ non- $\perp$ values, $(k+1) W$ distinct processes have contributed to $A U X[r]$, i.e., have SMV-broadcast PHASE $[r, 1]()$ messages. As $(k+1) W>n$, this is impossible.

$\square$ Lemma 2

Lemma 3. If $|E S T[r]| \leq k$, any correct process that starts round $r$ decides during $r$ a value of $E S T[r]$.

Proof As by assumption the correct processes have at most $k$ different estimate values at the beginning of round $r$, it follows from the SMV-Contribution property of the SMV-broadcast of line 4 that at least $(n-t)$ different processes contributed to the multiset view $w_{i}[r, 1]$. As $n-t \geq(W-1) k+1$ (algoritmic liveness), it follows that the multiset view $w_{i}[r, 1]$ of any correct process $p_{i}$ contains at least $W$ copies of a 
value of $E S T[r]$. Hence, $a u x_{i} \in E S T[r]$ at each correct process. Consequently $A U X[r] \subseteq E S T[r]$. it then follows that the predicate of line 7 is satisfied at any correct process $p_{i}$, which decides accordingly a value of view $w_{i}[r, 2] \subseteq A U X[r] \subseteq E S T[r]$, which concludes the proof of the lemma. $\quad \square_{\text {Lemma } 3}$

Lemma 4. Let $p_{i}$ and $p_{j}$ be two correct processes. At any round $r$, the predicates $\perp \notin v i e w_{i}[r, 2]$ and view $_{j}[r, 2]=\{\perp\}$ are mutually exclusive.

Proof let us assume by contradiction that $p_{i}$ is a correct process such that the predicate $\perp \notin$ view $i[r, 2]$ is satisfied (line 7), and $p_{j}$ a correct process such that the predicate view $w_{j}[r, 2]=\{\perp\}$ is satisfied (line 10).

It follows from the SMV-Contribution property of the SMV-broadcast issued by ${ }_{i}$ and ${ }_{j}$ at line 6 that $v_{i e w_{i}}[r, 2]$ contains values contributed by at least $(n-t)$ processes, and similarly for the set view $_{j}[r, 2]$ of $p_{j}$. As $n>3 t$, the intersection of any two sets of $(n-t)$ processes contains at least $(t+1)$ processes, i.e., one correct process. It then follows that there is a correct process that contributed to both view $i r, 2]$ and view $_{j}[r, 2]$, from which we conclude that either view $w_{i}[r, 2]$ contains $\perp$, or view $w_{j}[r, 2]$ contains a non- $\perp$ estimate value.

Lemma 5. No more than $k$ different values are decided by the correct processes.

Proof Let $r$ be the first round during which correct processes decide. They decide at line 8. Due to Lemma 2, the set $A U X[r]$ contains at most $k$ non- $\perp$ values. Moreover, due to the SMV-broadcast issued by the correct processes at line 6 that we have view $w_{i}[r, 2] \subseteq A U X[r]$ at each correct process $p_{i}$. Hence, due to line 7, a process that decides during round $r$ can only decide a value of $A U X[r]$.

Let us now consider a correct process $p_{j}$ that proceeds to round $(r+1)$. Let $p_{i}$ be a process that decides at round $r$. It follows from Lemma 4 that the predicates $\perp \notin v i e w_{i}[r, 2]$ and view $_{j}[r, 2]=\{\perp\}$ are mutually exclusive. Consequently, $p_{j}$ executes line 9 before progressing to the next round. Hence, $p_{j}$ updated $e s t_{j}$ to a non- $\perp$ value of $v i e w_{j}[r, 2] \subseteq A U X[r]$ before progressing to the next round. It follows that the estimates of the correct processes progressing to the next round are non- $\perp$ values of $A U X[r]$. Hence, $E S T[r+1] \subseteq A U X[r] \backslash\{\perp\}$. It then follows from Lemma 3 that at most $k$ values are decided.

Lemma 6. No correct process blocks forever in a round.

Proof The proof is by contradiction. Let $r$ be the first round at which a correct process $p_{i}$ blocks forever. It can block at line 4 or line 6 . Let us first consider line 4 . As no correct process blocked forever at a round $r^{\prime}<r$, all correct processes start round $r$ and invoke SMV_broadcast PHASE $[r, 1](-)$. It then follows from the MV-termination property that $p_{i}$ returns from its invocation. The same reasoning applies to line 6 , which concludes the proof of the lemma.

$\square$ Lemma 6

Lemma 7. If a correct process decides during a round $r$, any other correct process that does not decide by round $r$, decides during the round $(r+1)$.

Proof The proof is by contradiction. Let us suppose that a correct process $p_{i}$ decides $v$ at round $r$ (line 8 ) and a correct process $p_{j}$, which does not decide by round $r$. Due to Lemma $6, p_{j}$ proceeds to round $(r+1)$. Due to to Lemma 4 and the fact that $p_{i}$ decides at round $r$, it follows that view $w_{j}[r, 2] \neq\{\perp\}$. Hence, $p_{j}$ executes line 9, an assigns a non- $\perp$ of $A U X[r]$ to $e s t_{j}$. As $A U X[r]$ contains at most $k$ non- $\perp$ values (Lemma 2), we have $E S T[r+1] \subseteq A U X[r]$, i.e., the round $(r+1)$ starts with at most $k$ non- $\perp$ values. Due to to the Lemma $3, p_{j}$ decides in the round $r+1$. A contradiction. 
Lemma 8. Let VALID $[1,1]$ be the final (common) value of the sets mv_valid $d_{i}[1,1]$ of the correct processes. $\forall r$ we have $A U X[r] \subseteq V A L I D[1,1]$.

Proof The proof follows from the observation that the values, proposed by a correct process, which are not in $\operatorname{VALID}[1,1]$ can appear neither in view $i[r, 1]$ nor in view $i[r, 2]$. Hence, they cannot appear either in a set $A U X[r]$, and we have $A U X[r] \subseteq \operatorname{VALID}[1,1]$.

Lemma 9. All correct processes decide with probability 1.

Proof Due Lemma 7 if a correct process decides, all correct processes decides. Hence, let us assume by contradiction that no correct process decides.

Due to the MV-Equality property of the MV-broadcast generated by the invocations of SMV_broadcast PHASE $[1,1]()$ issued by the correct processes, there is a finite time $\tau$ after which the sets mv_valid $_{i}[1,1]$ of the correct processes remain forever non-empty and equal.

As no correct process blocks forever in a round (Lemma 6), all correct processes progress from round to round forever. Moreover, as the decision predicate of line 7 is never satisfied at a correct process, it follows that, after $\tau$, any correct process executes line 9 or line 10. Let us consider a round $r$ entered by all correct processes after time $\tau$. There are three cases.

- Case 1: At round $r$, all the correct processes execute line 9. So, each correct process sets its estimate to a non- $\perp$ value of $A U X[r]$. Due to Lemma 2, there are then at most $k$ different estimate (non- $\perp$ ) values in $A U X[r]$. Hence, all the correct processes start the round $(r+1)$, and $E S T[r+1]$ contains at most $k$ different estimate values (none being $\perp$ ). It then follows from Lemma 3 that all correct processes decide.

- Case 2: During $r$ at least one process (but not all) executes line 9. In this case, due to Lemma 2, each correct process $p_{i}$ that executes line 9 sets its current estimate $e s t_{i}$ to a non- $\perp$ value taken from the set $A U X[r]$, which contains at most $k$ non- $\perp$ values. The other processes execute line 10. This means that each of these processes ${ }_{i}$ sets its estimate value est $_{i}$ to a value $\in$ mv_valid $_{i}[r, 1]=$ $V A L I D[1,1]$. As $A U X[r] \subseteq V A L I D[1,1]$ (Lemma 8), there is a probability $p_{1}>0$ that they obtain values from $A U X[r]$.

- Case 3: During $r$ no process executes line 9. In this case, all the processes execute line 10. There is a probability $p_{2}>0$ that they obtain at most $k$ different estimate values.

In Case 1, all correct processes decide. Let us consider Case 2 and Case 3. During any round after $\tau$, there is a probability $p=\min \left(p_{1}, p_{2}\right)$ that the correct processes have at most $k$ different estimate values. Hence, there is a probability $P(\alpha)=p+p(1-p)+p(1-p)^{2}+\ldots+p(1-p)^{\alpha-1}=1-(1-p)^{\alpha}$ that, after at most $\alpha$ rounds, the processes have no more than $k$ estimate values. As $\lim _{\alpha \rightarrow \infty} P(\alpha)=1$, it follows that, with probability 1 , all processes will start a round with no more than $k$ estimate values. Then, according to Lemma 3, they will decide.

$\square_{\text {Lemma }} 9$

Theorem 4. Algorithm 4 solves the randomized Byzantine $k$-set agreement problem in the system model $\mathcal{B A M P}_{n, t}[t<n /(m+1), \mathrm{LRC}]$.

Proof The KS-Validity property follows from Lemma 1. The KS-Agreement property follows from Lemma 5. The KS-RR-Termination follows from Lemma 9.

$\square_{\text {Theorem } 4}$

\section{Conclusion}

This paper presented a signature-free randomized distributed algorithm that solves $k$-set agreement in asynchronous message-passing systems where up to $t \geq k$ processes may commit Byzantine failures. 
Its design is based on a modular construction which rests on (i) a broadcast abstraction which guarantees that two non-faulty processes cannot receive distinct messages from the same (possibly Byzantine) sender, and (ii) the stacking of two all-to-all communication abstractions which generalize the "binary" communication abstractions introduced in [14] to the multivalue domain. An interesting feature of the propsed algorithm lies in the validity condition it ensures, namely, no value proposed only by Byzantine processes can be decided by non-faulty processes.

\section{Acknowledgments}

This work has been partially supported by the French ANR project DISPLEXITY devoted to computability and complexity in distributed computing, and the Franco-German project DISCMAT devoted to the mathematics of distributed computing.

\section{References}

[1] Attiya H. and Welch J., Distributed computing: fundamentals, simulations and advanced topics, (2d Edition), WileyInterscience, 414 pages, 2004.

[2] Borowsky E. and Gafni E., Generalized FLP Impossibility Results for $t$-Resilient Asynchronous Computations. Proc. 25th ACM Symposium on Theory of Computing (STOC'93), ACM Press, pp. 91-100, 1993.

[3] Bouzid Z., Mostéfaoui A., and Raynal M., Minimal synchrony for Byzantine consensus. Proc. 34th ACM Symposium on Principles of Distributed Computing (PODC'15), ACM Press, 2015.

[4] Bracha G., Asynchronous Byzantine agreement protocols. Information \& Computation, 75(2):130-143, 1987.

[5] Censor Hillel K., Multi-sided shared coins and randomized set agreement. Proc. 22nd ACM Symposium on Parallelism in Algorithms and Architectures (SPAA'10), ACM Press, pp. 60-68, 2010.

[6] Chaudhuri S., More choices allow more faults: Set consensus problems in totally asynchronous systems. Information and Computation, 105(1):132158, 1993.

[7] Fischer M.J., Lynch N.A., and Paterson M.S., Impossibility of distributed consensus with one faulty process. Journal of the ACM, 32(2):374-382, 1985.

[8] Friedman R., Mostéfaoui A., and Raynal M., Simple and efficient oracle-based consensus protocols for asynchronous Byzantine systems. IEEE Transactions on Dependable and Secure Computing, 2(1):46-56, 2005.

[9] Gafni E. and Guerraoui R., Generalizing universality. Proc. 22nd Int'l Conference on Concurrency Theory (CONCUR'11), Springer LNCS 6901, pp. 17-27, 2011.

[10] Herlihy M.P., Kozlov D., and Rajsbaum S., Distributed computing through combinatorial topology, Morgan Kaufmann/Elsevier, 336 pages, 2014.

[11] Herlihy M., Shavit N., The Topological Structure of Asynchronous Computability. Journal of the ACM, 46(6):858-923, 1999.

[12] Lamport L., Shostack R., and Pease M., The Byzantine generals problem. ACM Transactions on Programming Languages and Systems, 4(3)-382-401, 1982.

[13] Lynch N.A., Distributed algorithms. Morgan Kaufmann Pub., San Francisco (CA), 872 pages, 1996 (ISBN 1-55860384-4).

[14] Mostéfaoui A., Moumen H., and Raynal M., Signature-free asynchronous binary Byzantine consensus with $t<n / 3$, $O\left(n^{2}\right)$ messages, and $O(1)$ expected time. Journal of the ACM, To appear, 2015.

[15] Mostéfaoui A. and Raynal M., Randomized k-set agreement. Proc. 12nd ACM Symposium on Parallelism in Algorithms and Architectures (SPAA'O0), ACM Press, pp. 291-297, 2000. 
[16] Mostéfaoui A. and Raynal M., Signature-free broadcast-based intrusion tolerance: never decide a Byzantine value. Proc. 14th Int'l Conference On Principles Of Distributed Systems (OPODIS'10), Springer LNCS 6490, pp. 144-159, 2010.

[17] Pease M., R. Shostak R., and Lamport L., Reaching agreement in the presence of faults. J. of the ACM, 27:228-234, 1980.

[18] de Prisco R., Malkhi D., and Reiter M.K., On $k$-Set consensus problems in asynchronous systems. IEEE Transactions on Parallel Distributed Systems, 12(1):7-21, 2001.

[19] Rabin M., Randomized Byzantine generals. Proc. 24th IEEE Symposium on Foundations of Computer Science (FOCS'83), IEEE Computer Society Press, pp. 116-124, 1983.

[20] Raynal M., Fault-tolerant agreement in synchronous message-passing systems. Morgan \& Claypool, 165 pages, 2010 (ISBN 978-1-60845-525-6).

[21] Raynal M., Concurrent programming: algorithms, principles and foundations. Springer, 515 pages, 2013 (ISBN 978-3642-32026-2).

[22] Raynal M., Stainer J., and Taubenfeld G., Distributed universality. Proc. 18th Int'l Conference on Principles of Distributed Systems (OPODIS'14), Springer LNCS 8878, pp. 469-484, 2014.

[23] Saks M. and Zaharoglou F., Wait-Free $k$-Set Agreement is Impossible: The Topology of Public Knowledge. SIAM Journal on Computing, 29(5):1449-1483, 2000.

[24] Srikanth T.K. and Toueg S., Simulating authenticated broadcasts to derive simple fault-tolerant algorithms. Distributed Computing, 2:80-94, 1987.

[25] Toueg S., Randomized Byzantine agreement. Proc. 3rd Annual ACM Symposium on Principles of Distributed Computing (PODC'84), ACM Press, pp. 163-178, 1984. 